\title{
Liver transplantation in acute-on-chronic liver failure
}

\author{
Roger Williams $\mathbf{s}^{1,2}$ \\ ${ }^{I}$ Institute of Hepatology London, Foundation for Liver Research, London SE5 9NT, UK. \\ ${ }^{2}$ Faculty of Life Sciences \& Medicine, King's College London, London SE5 9NT, UK.
}

Correspondence to: Prof. Roger Williams, Institute of Hepatology London, Foundation for Liver Research, 111 Coldharbour Lane, London SE5 9NT, UK. E-mail: r.williams@researchinliver.org.uk

How to cite this article: Williams R. Liver transplantation in acute-on-chronic liver failure. Hepatoma Res 2017;3:90-4.

Article history: Received: 31-03-2017 Accepted: 07-04-2017 Published: 17-05-2017

Over the past five years, we have gained much new knowledge of the cirrhotic patient with liver failure, sick enough to require admission to hospital. In Europe, this has come from the outstanding work of the Chronic Liver Failure (CLIF) Consortium set up by Rajiv Jalan, Vicente Arroyo, and other leading hepatologists to which I will be mainly referring to.$^{[1]}$ Shiv Sarin and colleagues in Asia Pacific despite using somewhat different definitions have reported similar findings and for their latest views on acute-onchronic liver failure (ACLF). I would refer you to the excellent review of Sarin and Choudhury ${ }^{[2]}$ in Nature Reviews Gastroenterology \& Hepatology, published in 2016. The characterisation of a syndrome of ACLF with defined subgroups has led to an improved prognostic assessment and provides a new basis for determining selection criteria for liver transplantation (LT) and of measures to enhance recovery from ACLF.

Some background first, on the massive clinical problem that hepatology faces from liver failure in Europe: 170,000 European citizens, it is estimated, die of cirrhosis each year - the 5th most common cause of death in individuals aged 45-65 years. Clinical decompensation heralded by ascites, hepatic encephalopathy, gastrointestinal bleeding or bacterial infection develops in more than $50 \%$ of patients within 10 years of the diagnosis of cirrhosis. Most importantly, there is a dramatic worsening of prognosis when this leads to involvement of other organs - multi organ failure (MOF).

Data from a French study as recently as 2014 illustrates how poor the outcome has been and remains so in many hospitals throughout the world for cirrhotic patients treated in the intensive care unit (ICU) and requiring ventilator support. ${ }^{[3]} \mathrm{A}$ third only of 246 consecutive patients became well enough to be discharged from the ICU and of these less than a half were alive at 1 year giving a $11 \%$ overall survival, 10 of the 27 survivors having had a liver transplant. The factors found to identify the risk of death after discharge, are measures of severity of the liver damage illness - bilirubin level, high Model for Endstage Liver Disease (MELD) score, on ventilator for $>9$ days. Almost all of the patients in this study as they had respiratory failure will have had other organ involvement bringing them within the designation of ACLF.

The characterisation of ACLF by the CLIF Consortium was based on data from the EASL-CLIF Acute-

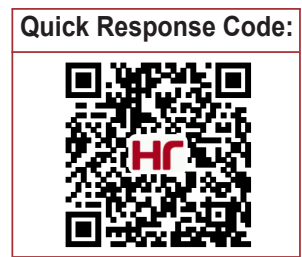



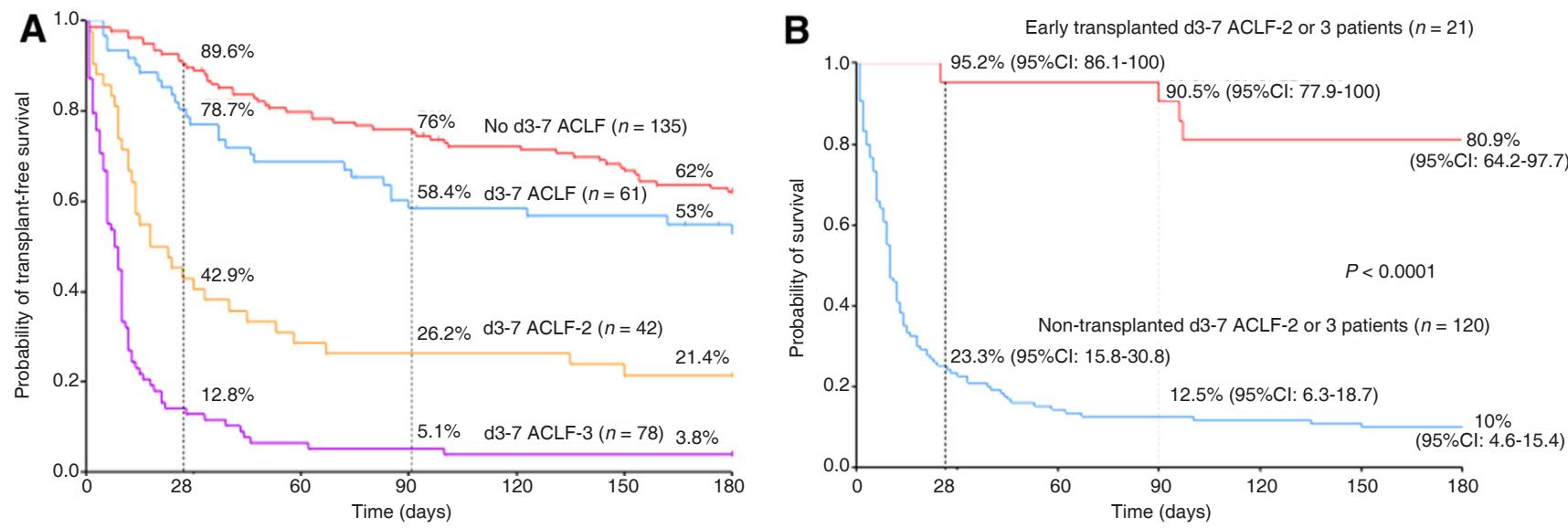

Figure 1: (A) Kaplan-Meier's 180-day transplant-free survival curves of patients based on their acute-on-chronic liver failure (ACLF) Grade at days 3-7 (d3-7 ACLF); (B) probability (180-day) of survival in patients with d3-7 ACLF-2 or -3 not transplanted and in patients undergoing early (28-day) liver transplantation. Kaplan-Meier's curves were compared using log-rank test. (Copyright Permission: Copyright (C) 2015 by the American Association for the Study of Liver Diseases. Gustot et al. Clinical Course of Acute-on-Chronic Liver Failure Syndrome and Effects on Prognosis. Hepatology. Publisher: Wiley)

on-Chronic Liver Failure in Cirrhosis (CANONIC) multicentre study of more than 1,300 patients with liver failure from cirrhosis admitted to 29 European hospitals. The subject of an excellent symposium published in the May 2016 issue of Seminars in Liver Disease with Rajiv Jalan as Guest Editor. ${ }^{[1]}$ ACLF is marked by rapid deterioration in liver function in a previously compensated or decompensated cirrhotic patient is accompanied by 1 or more other organ failures - kidney, brain, circulation, lungs and coagulation. Short-term mortality is high, more than $15 \%$ at 28 days. There is often a precipitating factor most frequently an exacerbation of liver damage from alcohol excess or HBV reactivation or the effects indirectly on the liver of a variceal bleed or infection. Interestingly, in $40 \%$ of cases no clear precipitating factor is identified. ACLF is to be distinguished clinically from acute decompensation in cirrhosis, with similar precipitating factors but which does not lead to failure of other organs apart from that of the liver and some form of non-kidney organ failure, and which has very much better overall prognosis with a $<5 \%$ mortality figure. Inflammation and the systemic inflammatory reaction is the driving force in the underlying pathophysiology as further indicated by high white cell and C-reactive protein levels.

It is important to take note of the dynamic nature of ACLF as evidenced by the findings of the CANONIC Study. With ACLF Grade 1 defined by 1 organ failure and mild renal impairment, over $50 \%$ of the cases resolve or improve. But with higher grades particularly Grade 3 when there are 3 or more organ failures, the percentage showing improvement is much lower $(16 \%) .{ }^{[4]}$ These figures give some indication of the scope for LT in ACLF. Changes in clinical status occur rapidly in ACLF and relevant to the consideration of $L T$ is the observation that the final clinical grade is usually reached by day 7 and at that time the prognosis in the individual case can be reliably predicted.

The development of scoring systems for the quantitation of prognosis in ACLF and for acute decompensation without MOF represent a major step forward. The CLIF-ACLF prognostic score is based on the CLIF organ failure score for 3 categories of severity for the 6 potential organ failures, namely, liver, kidney, brain, coagulation, circulation and respiration is combined with age and the white cell count as independent predictors of outcome. ${ }^{[5]}$ The scoring ranges from 0 to 100 points. ACLF scores have been shown to have superior prognostic accuracy compared to MELD and other commonly used scores as a result of capturing the markers of inflammation so important in the pathophysiology of the syndrome in addition to the quantitative assessment of organ failure severity. The probability of death for an individual patient at any one time can be determined by calculation of the equation, using an app or through the CLIF Consortium website.

The major influence of the ACLF grade at days 3-7 in determining prognosis by the transplant free survival curve [Figure 1]. The top 2 curves comprising patients with single organ failures and normal or raised serum creatinine values; $62 \%$ and $53 \%$ are alive at 180 days. Whereas for grade 2 and 3 ACLF survival figures at 180 days are considerably reduced at $21.4 \%$ and $3.8 \%$ respectively. The other half of the figure shows how well patients with grade 2 or 3 ACLF can do when transplanted; $80.9 \%$ of the cohort of 35 patients transplanted within 28 days of diagnosis alive at 180 days and with little fall off in survival at 1 year $(77 \%) .{ }^{[4]}$ 
Excellent survival results in those receiving a liver graft were also shown in the series reported by Finkenstedt et al. ${ }^{[6]}$ from centres in Austria of 144 patients fulfilling ACLF criteria of which 94 (65\%) were evaluated and $71(49 \%)$ listed for a transplant. One- and five-year survival figures for the $32(23 \%)$ patients transplanted were $87 \%$ and $82 \%$ respectively. Less than half of those who had got to the stage of being listed underwent transplantation and deaths on the waiting list were unacceptably high at $50 \%$ a measure of the very short period of time available for these sick patients to obtain a donor organ. Only $10(7 \%)$ of 144 patients in this series survived without a transplant - a similar figure to that for the French series of patients requiring mechanical ventilation shown earlier. At present ACLF is not considered an indication for priority or high urgency organ allocation despite the good outcomes that can be obtained.

An important question to ask is whether some of the deaths on the waiting list could have been prevented by the use of extra corporeal liver support devices, thereby giving more time for an organ to be obtained and allowing more patients in the grade 2 to 3 categories to be considered for LT. Currently the answer has to be "no". With the extracorporeal liver assist device containing a module of cultured hepatocytes (hepatoblastoma cell clone) providing synthetic and detoxifying functions in addition to toxin removal, survival as compared to the control group was improved only in those with a MELD score less than 28 and an age of less than 40 years, indicative perhaps of the potential for regeneration in this group 68.6 vs. 53.6 in controls $(P=0.077)$. In the major molecular adsorbents recirculating system trial of albumin dialysis, there was also no significant benefit overall with figures of $60.7 \%$ and $58.9 \%$ at 28 days for the treated and control groups despite improvement in some of the organ failures, namely, hepatic encephalopathy and circulatory disturbances. ${ }^{[7]}$ Possible reasons for this include the failure to correct the systemic reaction which is such an important part of the underlying pathophysiology of ACLF. Furthermore, in neither of the trials were the inclusion criteria based on CLIF diagnostic criteria and scoring. Of the new devices currently under clinical trial, one is based on membrane absorption of endotoxin from the circulation, the other has a more powerful microporous charcoal as the absorbent.

Turning to plasma exchange which is widely used in the Far East for the commonest form of ACLF, namely, hepatitis $B$ reactivation. Replacement of the patients' plasma with its wide range of toxins and mediators by fresh frozen plasma is thought to facilitate liver regeneration and recovery. In each of the three studies summarised [Table 1], ${ }^{[8-10]}$ there was a statistically significant improvement either in survival or in the obtaining of a reduced MELD score prior to LT. I would ask you also to take note of the very recently published study of high volume plasma exchange in acute liver failure (ALF) showing in those not transplanted, significant survival improvement. ${ }^{[11]}$ In ALF, the underling dysfunctional immune reaction responsible for the multi-organ failure and susceptibility to sepsis is very similar to that which has been demonstrated in ACLF.

It is relevant also in the context of LT for ACLF to mention a number of therapeutic measures which may enhance resolution and improve the survival of patients with hepatic decompensation. These include reduction in bacterial translocation with rifaximin or probiotics and oral carbon for absorption of toxic bacterial products. Currently under clinical trial also is IV human serum albumin with its wide range of antioxidant and immunomodulatory effects. Enhancing liver hepatic regeneration through administration of G-CsF is another approach and there is some experience of this use in ACLF. The mechanism is thought to be mobilisation of hematopoietic stem cells to the liver leading to an increased number of CD34 positive progenitor cells stimulating the regeneration process. This was demonstrated in the study of Garg et al. ${ }^{[12]}$ leading to a significant survival benefit. Duan et al. ${ }^{[13]}$ also reported improved survival at 90 days, in ACLF from HBV reactivation in association with a rise in peripheral neutrophil and CD34 positive cell counts. Sarin and Choudhury ${ }^{[2]}$ from New Delhi have pioneered this exciting new therapeutic approach and more details of later studies are in the reference I gave at the beginning of this presentation.

In addition to the CLIF scoring, a number of biomarkers reflecting the severity of liver injury and of multi-organ failure have been identified which may add to prognostic information of the ACLF score and may be of particular value in early diagnosis and in assessing progression. Hyponatremia has been shown to have an independent predictive effect on 90 days survival and plasma copeptin reflecting changes in vasopressin level have been shown to improve

Table 1: Value of plasma exchange widely used in Far East for ACLF from HBV reactivation

\begin{tabular}{lc}
\hline Studies & Changes \\
\hline Mao et al. ${ }^{[8]}(2010)$ & 30 days survival $50 \%$ vs. $31.7 \%$ \\
Ling et al. ${ }^{[9]}(2012)$ & Reduced MELD prior to LT \\
Wan et al. ${ }^{[10]}(2015)$ & 12 weeks survival $29 \%$ vs. $14 \%$ \\
\hline
\end{tabular}

ACLF: acute-on-chronic liver failure; HBV: hepatitis B virus; MELD: Model for End-stage Liver Disease; LT: liver transplantation 
performance of the ACLF score. A very recent 2016 publication showed increased values for urinary neutrophil gelatinase-associated lipocalin (N-GAL) related to stage and severity of cirrhosis as another independent biomarker of ACLF prognosis. ${ }^{[14]} \mathrm{N}-\mathrm{GAL}$ is the product of up regulation of the $\mathrm{cn} 2$ gene in the liver driven by the processes of liver cell destruction.

Finally, to return to the selection and prioritisation of patients for transplantation in the clinical setting. Rajiv Jalan, Royal Free Hospital, London, suggests that ACLF scores of up to 30 are consistent with spontaneous recovery and patients should have serial assessments on a regular basis to determine whether this is occurring. With a 30 to 65 score, the patient is unlikely to survive without a transplant and depending on co-morbidity and other criteria should be given priority for an urgent transplant in anticipation of excellent results. A score above 65 raises questions of futility and withdrawal of active treatment measures. The subject is considered in some depth in a recent paper from Putignano and Gustot. ${ }^{[15]}$

In summary, 3 points relating to transplantation in ACLF: firstly, improvement or worsening in ACLF grade occur rapidly and likely survival is best predicted at 3-7 days; secondly, transplantation gives good results in those with deteriorating ACLF grades 2 to 3 but timing, priority and selection criteria need to be defined; thirdly, liver support devices, plasma exchange, anti-inflammatory agents and stimulation of regeneration require further evaluation.

\section{Authors' contributions}

R. Williams contributed solely.

\section{Financial support and sponsorship} None.

\section{Conflicts of interest}

There are no conflicts of interest.

\section{Patient consent}

Not applicable.

\section{Ethics approval}

Not applicable.

\section{REFERENCES}

1. Jalan R, Moreau R, Kamath PS, Arroyo V. Acute-on-chronic liver failure: a distinct clinical condition. Semin Liver Dis 2016;36:107-8.

2. Sarin SK, Choudhury A. Acute-on-chronic liver failure: terminology, mechanisms and management. Nat Rev Gastroenterol Hepatol 2016;13:131-49.

3. Levesque E, Saliba F, Ichaï P, Samuel D. Outcome of patients with cirrhosis requiring mechanical ventilation in ICU. $J$ Hepatol 2014;60:570-8

4. Gustot T, Fernandez J, Garcia E, Morando F, Caraceni P, Alessandria C, Laleman W, Trebicka J, Elkrief L, Hopf C, SolísMunoz P, Saliba F, Zeuzem S, Albillos A, Benten D, MonteroAlvarez JL, Chivas MT, Concepción M, Córdoba J, McCormick A, Stauber R, Vogel W, de Gottardi A, Welzel TM, Domenicali M, Risso A, Wendon J, Deulofeu C, Angeli P, Durand F, Pavesi M, Gerbes A, Jalan R, Moreau R, Ginés P, Bernardi M, Arroyo V; CANONIC Study Investigators of the EASL-CLIF Consortium. Clinical course of acute-on-chronic liver failure syndrome and effects on prognosis. Hepatology 2015;62:243-52.

5. Jalan R, Saliba F, Pavesi M, Amoros A, Moreau R, Ginès $P$, Levesque E, Durand F, Angeli P, Caraceni P, Hopf C, Alessandria C, Rodriguez E, Solis-Muñoz P, Laleman W, Trebicka J, Zeuzem S, Gustot T, Mookerjee R, Elkrief L, Soriano G, Cordoba J, Morando F, Gerbes A, Agarwal B, Samuel D, Bernardi M, Arroyo V; CANONIC study investigators of the EASL-CLIF Consortium. Development and validation of a prognostic score to predict mortality in patients with acute-on-chronic liver failure. $J$ Hepatol 2014;61:1038-47.

6. Finkenstedt A, Nachbaur K, Zoller H, Joannidis M, Pratschke J, Graziadei IW, Vogel W. Acute-on-chronic liver failure: excellent outcomes after liver transplantation but high mortality on the wait list. Liver Transpl 2013;19:879-86.

7. Bañares R, Nevens F, Larsen FS, Jalan R, Albillos A, Dollinger M, Saliba F, Sauerbruch T, Klammt S, Ockenga J, Pares A, Wendon J, Brünnler T, Kramer L, Mathurin P, de la Mata M, Gasbarrini A, Müllhaupt B, Wilmer A, Laleman W, Eefsen M, Sen S, Zipprich A, Tenorio T, Pavesi M, Schmidt HH, Mitzner S, Williams R, Arroyo V; RELIEF study group. Extracorporeal albumin dialysis with the molecular adsorbent recirculating system in acute-on-chronic liver failure: the RELIEF trial. Hepatology 2013;57:1153-62.

8. Mao W, Ye B, Lin S, Fu Y, Chen Y, Chen Y. Prediction value of model for end-stage liver disease scoring system on prognosis in the acute on chronic liver failure patients with plasma exchange treatment. ASAIO J 2010;56:475-8.

9. Ling Q, Xu X, Wei Q, Liu X, Guo H, Zhuang L, Chen J, Xia Q, Xie H, Wu J, Zheng S, Li L. Downgrading MELD improves the outcomes after liver transplantation in patients with acute-onchronic hepatitis B liver failure. PLoS One 2012;7:e30322.

10. Wan YM, Yang LH, Yang JH, Xu Y, Yang J, Song GB. The effect of plasma exchange on entecavir-treated chronic hepatitis B patients with hepatic de-compensation and acute-on-chronic liver failure. Hepatol Int 2015;10:462-9.

11. Larsen FS, Schmidt LE, Bernsmeier C, Rasmussen A, Isoniemi H, Patel VC, Triantafyllou E, Bernal W, Auzinger G, Shawcross D, Eefsen M, Bjerring PN, Clemmesen JO, Hockerstedt K, Frederiksen HJ, Hansen BA, Antoniades CG, Wendon J. High-volume plasma exchange in patients with acute liver failure: an open randomised controlled trial. J Hepatol 2016;64:69-78.

12. Garg V, Garg H, Khan A, Trehanpati N, Kumar A, Sharma BC, Sakhuja P, Sarin SK. Granulocyte colony-stimulating factor mobilizes CD34(+) cells and improves survival of patients with acute-on-chronic liver failure. Gastroenterology 2012;142:505-12. e1.

13. Duan XZ, Liu FF, Tong JJ, Yang HZ, Chen J, Liu XY, Mao YL, Xin SJ, Hu JH. Granulocyte-colony stimulating factor therapy improves survival in patients with hepatitis B virus-associated acuteon-chronic liver failure. World J Gastroenterol 2013;19:1104-10. 
14. Ariza X, Graupera I, Coll M, Solà E, Barreto R, García E, Moreira R, Elia C, Morales-Ruiz M, Llopis M, Huelin P, Solé C, Fabrellas N, Weiss E, Nevens F, Gerbes A, Trebicka J, Saliba F, Fondevila C, Hernández-Gea V, Fernández J, Bernardi M, Arroyo V, Jiménez W, Deulofeu C, Pavesi M, Angeli P, Jalan R, Moreau R, SanchoBru P, Ginès P; CANONIC Investigators, EASL CLIF Consortium.
Neutrophil gelatinase-associated lipocalin is a biomarker of acuteon-chronic liver failure and prognosis in cirrhosis. $J$ Hepatol 2016;65:57-65.

15. Putignano A, Gustot T. New concepts in acute-on-chronic liver failure: implications for liver transplantation. Liver Transpl 2017;23:234-43 